\title{
USO DE LA ADMINISTRACIÓN DE OPERACIONES EN LAS MIPYMEs .
}

Luis Solís, Orlando Pérez, Ivonne Burgos, José Villao 


\title{
Uso de la Administración de Operaciones en las Mipymes Use of Operations Management in MSMEs
}

\author{
Luis-Eduardo Solís Granda ${ }^{1}$; Orlando Agustín Pérez Manzo루 Ivonne Soraya Burgos Villamar; José Fabián Villao \\ Viteri $^{2}$ \\ ${ }^{1}$ Universidad Estatal de Milagro (UNEMI); \\ luchosolisg@gmail.com \\ ${ }^{2}$ Universidad Estatal Península de Santa Elena (UPSE)
}

\begin{abstract}
Resumen
La operacionalización empleada por las micro-pequeñas y medianas empresas (Mipymes) se ha estudiado bajo diferentes enfoques, aplicando varios modelos estratégicos que involucran diferentes metodologías de administración con el fin de maximizar la productividad y por ende que se mejore el rendimiento de las operaciones, y de esta manera se vean beneficiadas todas las partes de la organización, sin embargo, aún no se ha definido la mejor herramienta para alcanzar este fin. En este sentido, este trabajo de investigación analiza el uso de la administración de operaciones, exponiendo los principales conceptos y herramientas que permitan mejorar el rendimiento de las Mipymes del cantón Milagro, Ecuador. La investigación tiene un enfoque cuantitativo. Los resultados obtenidos con una muestra de 192 empresas y la revisión literaria realizada permitieron identificar que no se maneja adecuadamente las áreas estratégicas de decisión afectando directamente al rendimiento de la empresa.
\end{abstract}

Palabras Claves: Administración de Operaciones, Mipymes, productividad, rendimiento.

\begin{abstract}
The operationalization used by SMEs has been studied under different approaches, applying several strategic models that involve different management methodologies in order to maximize production and therefore that the operations are benefited in a general way. However, the best tool to achieve this has not been defined. In this sense, this research analyzes the use of operations management, exposing the main concepts and tools that allow to improve the performance of SMEs in Milagro. The research has a quantitative approach. The results obtained with a sample of 192 companies showed that the strategical area of decision is not handled adequately affecting directly to the performance of the company.
\end{abstract}

Key Words: Operations Management, SMEs, performance, productivity. 


\section{Introducción}

Actualmente, la micro-pequeña y mediana empresa (mipymes) presenta una compleja evolución en un entorno de negocios caracterizado por la globalización de los mercados, esto origina la necesidad de incrementar su desempeño y requerir la implementación de estrategias que beneficien las operaciones internas y externas de la organización, todo con la finalidad de reducir los costos de operación, mejorar la eficiencia de los procesos, los niveles de inventario, la calidad de los productos y por supuesto incrementar la productividad.

La pyme no sólo aporta al crecimiento económico de un país a través de la generación de empleo como se cree habitualmente, sino que también contribuye cuantitativamente a la fundamentación económica de los mismos, además de la participación social que éstas generan [1]. En general la competitividad de las pymes latinoamericanas no sólo se enmarca en cuánto pueden producir en términos monetarios, sino que también están asociados a otros factores. Las dificultades que éstas pueden tener también se ven en gran aumento y no solo se pueden considerar factores internos sino también externos que imposibilitan el desarrollo óptimo de estas empresas.

La micro-pequeña y mediana empresa se considera un sector sumamente importante dentro de la economía de un país, puesto que impulsa la generación de empleo ayudando a armonizar los mercados, manteniendo una balanza entre la oferta y la demanda. Muchos países que ya han aceptado esta realidad realizan muchos esfuerzos para salvaguardar a estas organizaciones entre los que se pueden destacar el diseño de cuerpos legales que rijan exclusivamente a este tipo de empresas [2].

Las mipymes, deben enfrentar un sin número de situaciones dentro de su operatividad, las mismas que se encuentran relacionadas a la gestión de la empresa, el manejo de la información, la calidad de sus productos, la eficiencia de sus procesos, la competitividad del mercado, la obtención de financiamiento y los avances tecnológicos, muchos de estos aspectos ponen en desventaja a este tipo de organizaciones, sin embargo con el fenómeno de la globalización se han desprendido una gran cantidad de oportunidades dejándoles a estas una opción de que empleando buenas estrategias puedan subsistir en el mercado [3].

Esto hace que se genere la necesidad por parte de la micro pequeña y mediana empresa de buscar las mejores estrategias que les permitan seguir creciendo en el medio, logrando una excelente flexibilidad, la maximización de la productividad y el aumento de la rentabilidad de las operaciones. Evaluar el nivel de competitividad desde el análisis de toda la cadena de valor de la empresa, considerando que cada componente de la misma aporta al mejoramiento de los procesos de la empresa, para el empleo de una buena metodología que permita realizar un buen análisis se deben considerar todas las actividades y los recursos que posea la compañía de esta manera se podrá generar ventajas que le permitan ser más competitivas y sostenibles en el mercado [4].

En base a lo expresado en los párrafos anteriores se justifica la realización del articulo porque las mipymes se distinguen por ser rentables, generar empleos $\mathrm{y}$ contribuir a la economía de un país, por lo tanto, es necesario que las mismas logren triunfar y mantenerse en el mercado. La ciudad de Milagro es un cantón ubicado en la provincia del Guayas, que tiene un acelerado crecimiento y desarrollo económico, según datos del Sistema de Consultas (REDATAM, 2010) existen 4.954 empresas en este cantón, de las cuales resaltan con un mayor número las relacionadas a los sectores de la Agricultura, Comercio, Servicios e Industrias manufactureras.

Esta investigación presenta un análisis del uso de la administración de operaciones como una alternativa para las mipymes del cantón Milagro, cuya población es de 384 empresas. A partir de la siguiente sección se define la administración de operaciones, los principales componentes que se deben conocer y además se exponen estudios realizados sobre esta temática, posterior a ello se define la metodología, se analizan los resultados y, finalmente, se exponen las conclusiones de la investigación.

\section{Desarrollo}

La administración de operaciones posee un campo muy amplio de aplicación dentro de las empresas y por supuesto está netamente ligada a elevar la productividad de las mismas, los gerentes de operaciones son los encargados de realizar el análisis de las funciones de operaciones y la relación de ésta con las demás funciones de la empresa. Es importante que tengan diseñado mecanismos de control y una buena planificación para todo el sistema de producción con la finalidad de salvaguardar los intereses dentro de la organización y la relación con el ambiente externo [5].

En torno a esto se considera importante definir el concepto de la administración de operaciones para el entendimiento de lo que se quiere lograr a través de esta investigación, de forma simplificada, algunos la definen como una estrategia que permite realizar una buena planeación de los recursos de la empresa apoyando de esta forma a la mejora continua de los procesos.

Existen otros conceptos más estructurados, la administración de operaciones es una agrupación de actividades que crean valor al transformar los insumos en productos terminados a través de la producción de bienes o servicios, estas actividades son realizadas por todas las organizaciones [6]. En las empresas manufactureras evidentemente se estaría hablando de producción de bienes, en estas podemos ver la creación de un producto tangible.

La administración de operaciones es la disciplina que estudia la planeación, organización, dirección y 
control de las operaciones productivas, actividades necesarias para producir los bienes y servicios que ofrecen las empresas y las organizaciones dedicadas a la producción de manufacturas y servicios [7]. Sin embargo, no basta con definir la administración de operaciones para tener una idea clara de lo que sucede en las mipymes en cuanto a su productividad, pero sí permite conocer la importancia que tiene la existencia de un administrador de operaciones dentro de una organización.

Con esto se puede prevenir cualquier anomalía en la cadena de valor de la empresa que interrumpa el buen funcionamiento de los procesos de producción. La administración de operaciones debe hacer frente a diez decisiones estratégicas, que son: Diseño de Bienes y Servicios, administración de la Calidad, Diseño del proceso y la capacidad, Estrategias de Localización, Estrategias de distribución de instalaciones, Recursos Humanos y diseño del trabajo, administración de la cadena de suministros, Inventario, Programación a mediano y corto plazo, Mantenimiento [6]. La Tabla 1 muestra una descripción de cada una de las Decisiones Estratégicas.

Tabla 1. Diez Decisiones críticas en la Administración de Operaciones

\begin{tabular}{|c|c|}
\hline Áreas de Decisión & Enfoque desde el punto de vista de la Investigación \\
\hline Bienes y Servicios & $\begin{array}{l}\text { En esta decisión el Administrador debe ponerse en el lugar del cliente para } \\
\text { analizar que bienes o servicios son necesarios o cuales se podrían mejorar } \\
\text { de los ya existentes y plantearse, ¿Cuál sería la mejor manera de diseñarlos? }\end{array}$ \\
\hline Calidad & $\begin{array}{l}\text { Asegurar la calidad es un punto crítico en el proceso de producción, la } \\
\text { empresa debe tener buenos estándares y políticas definidas que le permitan } \\
\text { al administrador realizar un control minucioso desde la elaboración del bien } \\
\text { y servicio hasta la supervisión del personal responsable de la calidad de los } \\
\text { mismos. }\end{array}$ \\
\hline Proceso y Capacidad & $\begin{array}{l}\text { En esta etapa se debe conocer, cuáles son los requerimientos técnicos, } \\
\text { tecnológicos y humanos que se requieran para la elaboración de los } \\
\text { productos }\end{array}$ \\
\hline Localización & $\begin{array}{l}\text { Tanto oficinas administrativas como bodegas o centros de distribución } \\
\text { deben tener una justificación de su localización, se deben plantear criterios } \\
\text { en los que se base un estudio de mercado para conocer, cuál sería la mejor } \\
\text { ubicación para las mismas. }\end{array}$ \\
\hline $\begin{array}{l}\text { Organización, Distribución e } \\
\text { Instalaciones }\end{array}$ & $\begin{array}{l}\text { ¿Cuán grande debe ser la organización?, para cumplir con los objetivos de } \\
\text { la empresa y así mismo la distribución de las instalaciones debe ser la más } \\
\text { acertada en función de la extensión que se desee lograr. }\end{array}$ \\
\hline Recursos Humanos & $\begin{array}{l}\text { En el Recurso Humano se deben considerar muchos factores tanto desde el } \\
\text { punto de vista de la organización como del empleado, se puede preguntar } \\
\text { que tanto debe esforzarse y producir un obrero, pero a su vez se debe } \\
\text { plantear, ¿Como la organización proporcionará un ambiente de trabajo } \\
\text { adecuado a ese empleado? }\end{array}$ \\
\hline Cadena de Suministro & $\begin{array}{l}\text { La organización debe asegurar que los proveedores con los que se cuentan } \\
\text { cumplan con lo estipulado para no caer en atraso de los procesos propios, } \\
\text { así mismo se debe definir cuáles son los componentes que se realizarán y } \\
\text { cuales se adquirirán de terceros, todo el enfoque se debe realizar en la visión } \\
\text { de satisfacer eficientemente las necesidades del cliente. }\end{array}$ \\
\hline Inventario & $\begin{array}{l}\text { El inventario es otro punto crítico de análisis en el que siempre se debe } \\
\text { prevenir situaciones, el administrador debe tener en claro ¿Cuál es la } \\
\text { capacidad de producción de la empresa? y ¿Cuál es el volumen de ventas de } \\
\text { la misma? Con esto podrá tener un control de los requerimientos que debe } \\
\text { hacer para la elaboración de los productos y a su vez poder cumplir con los } \\
\text { pedidos de los clientes. }\end{array}$ \\
\hline
\end{tabular}




\begin{tabular}{|l|l|}
\hline Programación & $\begin{array}{l}\text { La programación debe responder a varios sucesos a los que se enfrentan un } \\
\text { gerente o administrador, se debe analizar cuestiones como en que parte del } \\
\text { proceso se debe subcontratar, es necesario mantener al personal o realizar } \\
\text { contratos por períodos de producción entre otras situaciones. }\end{array}$ \\
\hline Mantenimiento & $\begin{array}{l}\text { Es indispensable para una organización que produce bienes el } \\
\text { mantenimiento de sus maquinarias y procesos de producción, se debe tener } \\
\text { en claro los responsables y los tiempos idóneos para los mantenimientos. }\end{array}$ \\
\hline
\end{tabular}

Fuente: Elaboración Propia.

Todas estas definiciones y descripciones demuestran que la implementación de la administración de operaciones agrega grandes beneficios al interés de la creación de valor de una empresa, sin embargo, las mipymes son empresas que se enfrentan a una competitividad muy agresiva impulsándolas a la constante implementación de nuevos conceptos en su organización. En este sentido se plantea que las mipymes se acojan a la administración de operaciones, la cual, como se puede observar tiene varias áreas de decisión que aportan en el desarrollo de la empresa. Sin embargo, [5] mencionan que la función de operaciones tiene bajo su responsabilidad cuatro áreas principales de decisión: los procesos, la calidad, la capacidad y el inventario. En la Figura 1 se relacionan las áreas detalladas en el párrafo anterior tratando de esta manera justificar la importancia de las mismas en la toma de decisiones de los gerentes de operaciones desde el punto de vista de varios autores [8], [9], [10], [11].

\section{CALIDAD}

Es garantía de sostenibilidad de la empresa, para su analisis se deben considerar diversos tipos con el el fin de alcanzar una calidad total, sin embargo, esta tambien depende de otros elementos

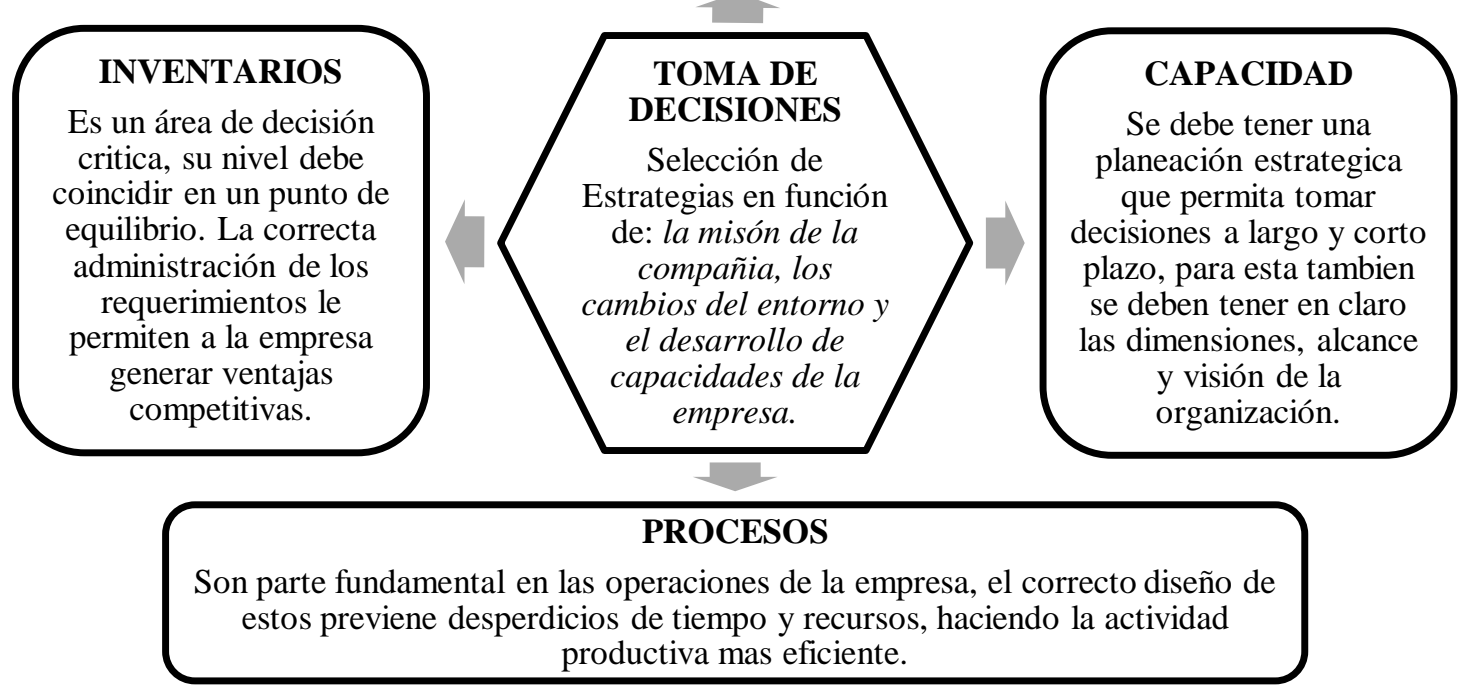

Figura 1. Principales áreas de decisión en la organización.

Se debe diseñar y ejecutar controles internos de operación para que la implementación de la administración de operaciones se la pueda realizar de forma eficiente en la organización, hay factores que están fuertemente combinados y que se deben estudiar cuidadosamente para lograr un rendimiento óptimo y que este pueda ser medido constantemente, estos factores ya han sido mencionados anteriormente, sin embargo, se vuelven a reseñar para concluir la idea de la reflexión, estos son: la planeación y control de la producción, gestión de suministros, inventarios y calidad, así como también el control del equipo de operaciones [12].

\section{Materiales y Métodos}

El trabajo que se expone a través del presente artículo involucró una investigación de campo. Se aplicó una encuesta dirigida a la población de aquellas micro-pequeñas y medianas empresas debidamente establecidas en el cantón Milagro. Según el INEC 
(Instituto Nacional de Estadísticas y Censos) ${ }^{\mathrm{I}}$ asciende a 4.954, comprendidas entre micro, pequeñas, medianas y grandes empresas pertenecientes a los Sectores Manufacturero, Sector de Comercio, Sector de Servicios y Otros (Agricultura, Minas, Organizaciones y Órganos Extraterritoriales).
De acuerdo con la unidad de análisis de la investigación se considera para efectos de la población únicamente a las Mipymes relacionadas al sector de Manufactura, que según el INEC son 384 en el Cantón Milagro, una vez estratificada la población dependiendo de los estratos de personal ocupado. La Tabla 2 muestra la población considerada para la investigación.

Tabla 2. Población estratificada según los estratos de personal ocupado

\begin{tabular}{|cccc|}
\hline $\begin{array}{c}\text { Estratos de personal } \\
\text { ocupado }\end{array}$ & $\begin{array}{c}\text { Total de empresas } \\
\text { manufactureras }\end{array}$ & Porcentaje & $\begin{array}{c}\text { n } \\
\text { (Muestra) }\end{array}$ \\
\hline $1-9$ & 379 & $98,70 \%$ & $\mathbf{1 8 9}$ \\
$10-49$ & 4 & $1,04 \%$ & $\mathbf{2}$ \\
$50-99$ & 0 & $0,00 \%$ & $\mathbf{0}$ \\
$100-199$ & 1 & $0,26 \%$ & $\mathbf{1}$ \\
\hline Total & $\mathbf{3 8 4}$ & $\mathbf{1 0 0 , 0 0 \%}$ & $\mathbf{1 9 2}$ \\
\hline
\end{tabular}

Fuente: Instituto Nacional de Estadísticas y Censos.

\subsection{Muestreo}

A partir de la fórmula para población finita:

$$
n=\frac{N p q}{\frac{(N-1) E^{2}}{Z^{2}}+p q}
$$

Con un nivel (Z) de confianza del 95\%, un error (E) del $5 \%$ y probabilidades (p y q) de 0.50 , se determinó un tamaño de muestra (n) de 192 mipymes a encuestar. La distribución de la muestra se realizó de forma proporcional (ver Tabla 2). Una vez definida la muestra, en la Tabla 3 se resumen los datos técnicos de la investigación propuesta.

Tabla 3. Ficha técnica de la investigación

\begin{tabular}{|ll|}
\hline Características & Encuesta \\
\hline Población & $\begin{array}{l}384 \text { micro pequeñas y medianas empresas manufactureras de la } \\
\text { zona }\end{array}$ \\
Ámbito de estudio & Cantón Milagro \\
Unidad muestral & Empresas de 1 a 199 trabajadores \\
Método de recolección de la información & Encuesta personal \\
Procedimiento de muestreo & Aleatorio simple \\
Margen de error de muestreo & $\pm 5 \%$ a un nivel de confianza del $95 \%$ \\
Fecha de trabajo de campo & Diciembre 2016 a marzo 2017 \\
\hline
\end{tabular}

Fuente: Elaboración Propia.

\section{Resultados}

La información obtenida, por parte de cada uno de los gerentes - propietarios de las mipymes de manufacturas establecidas en el Cantón Milagro, ha sido de vital importancia para la investigación, debido a que permite conocer qué grado de conocimientos tiene cada una de las empresas, sobre la aplicación de la administración de operaciones.

Al realizar la encuesta se revela que el 56.6\% de las Mipymes de manufacturas realizan planes de

${ }^{I}$ INSTITUTO NACIONAL DE ESTADISTICAS Y CENSOS:

Censo Nacional Económico 2010, redatam.inec.gob.ec. 
producción a mediano plazo. En lo referente a la programación de la producción se observa que el $31.3 \%$ la realiza sobre la marcha y un $12 \%$ diariamente, estos resultados muestran que existe alta variabilidad en la programación de la producción generando con esto inestabilidad en los procesos. Ver Figura 2.

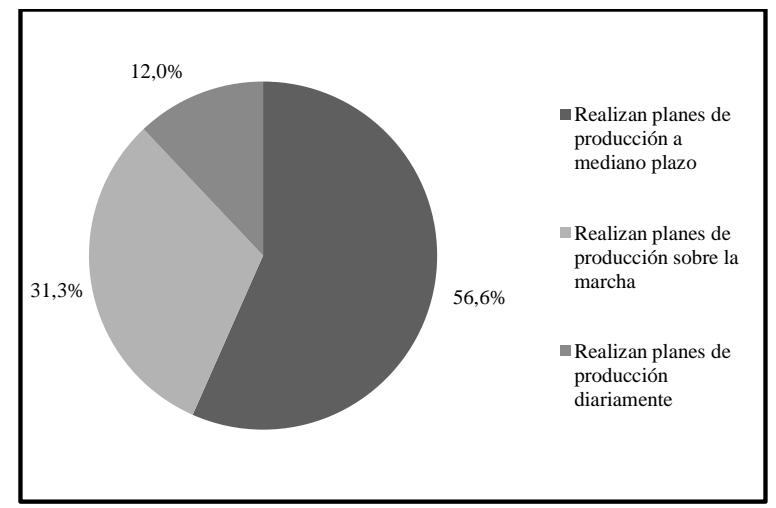

Figura 2. Planificación de la producción

Entre los resultados de las encuestas también se obtuvo el $93 \%$ de las empresas trabajan en un solo turno variable entre 8 y 10 horas diarias, mientras que un $7 \%$ de estas tienen horarios compuestos entre 11 y 12 horas de trabajo (Figura 3), este factor es importante a considerar debido que si los empleados trabajan demasiadas horas al día se disminuye la productividad de los mismos y por ende no se desarrollarán adecuadamente los procesos.

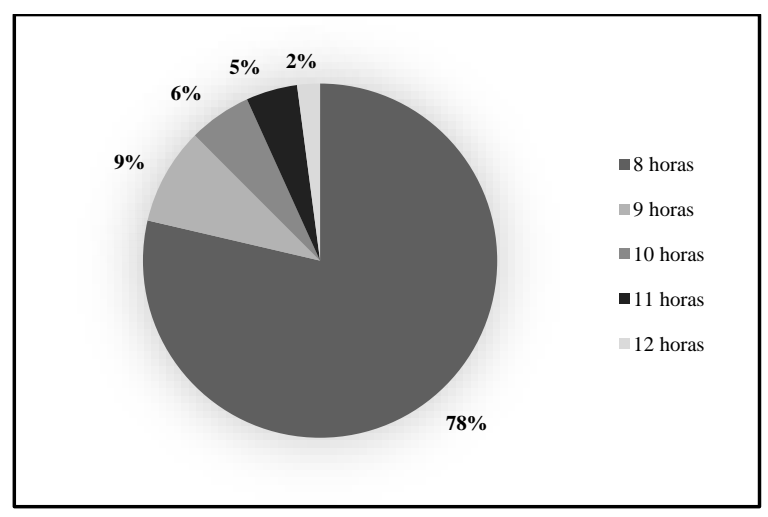

Figura 3. Horas diarias de trabajo

En lo que respecta a la operación de despacho de pedidos a clientes (Figura 4), los resultados de las encuestas nos permitieron descubrir que en un $79 \%$ de las empresas, esta tarda de 1 a 5 días, Otras empresas realizan su gestión de despacho en períodos menores, un $9 \%$ lo hace entre 1 y 4 días, el $7 \%$ tiene un tiempo estimado entre 1 y a 3 días mientras que un $5 \%$ despacha sus pedidos entre 1 a 2 días. Dialogando con los propietarios de las empresas se pudo conocer que entre las causas de tardanza en los despachos están los retrasos en la producción y el suministro de los insumos requeridos para el proceso.

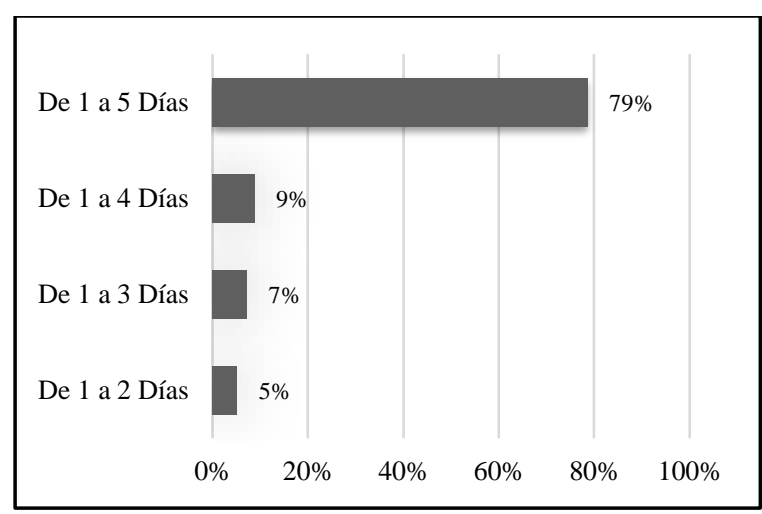

Figura 4. Tiempo (días) de despacho de pedidos

En referencia a la distribución (Figura 5), el estudio muestra que en su gran mayoría las mipymes utilizan el modo de transporte por carretera para distribuir sus productos y un reducido grupo de empresas utiliza el modo de transporte aéreo.

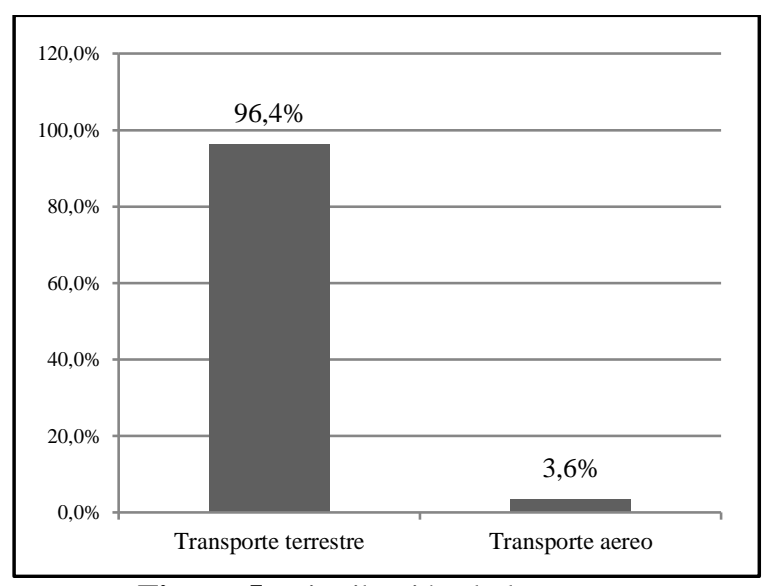

Figura 5. Distribución de las ventas

En lo concerniente a los inventarios los resultados de las encuestas permitieron descubrir que en un $80 \%$ de las empresas no cuentan con procedimientos adecuados en el manejo de sus inventarios en tanto que el $20 \%$ de las empresas manifiestan que si poseen un adecuado control en sus inventarios. Ver Figura 6.

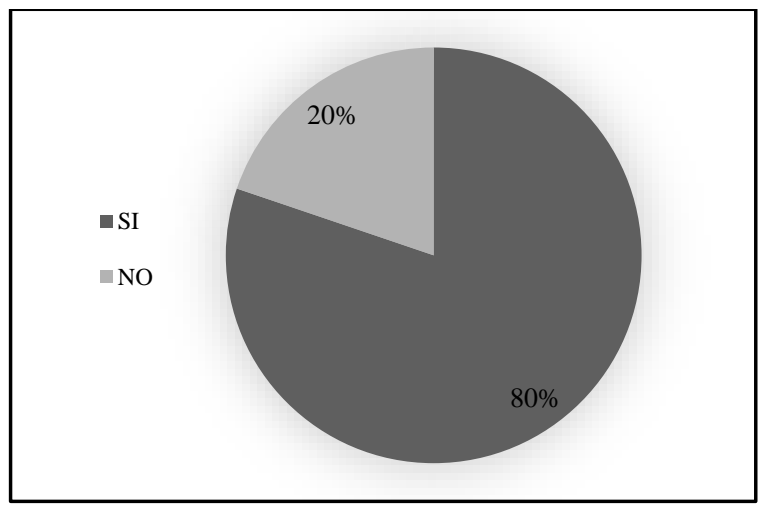

Figura 6. Procedimiento de Inventarios 


\section{Conclusiones}

Al analizar la situación actual de las Mipymes de manufactura de la ciudad de Milagro se ha encontrado que los gerentes propietarios enfrentan limitaciones que restringen su competitividad, para lo cual los gerentes deben capacitarse en lo relacionado al manejo de inventarios, optimización de los procesos a través de las técnicas de mejora continua, mejorar el control de los pronósticos de abastecimientos y la reducción de costos.

Los empresarios piensan que una empresa solo compite ofreciendo productos y/o servicios nuevos, lo cual es falso debido a que las empresas también compiten con la aplicación de políticas y directivas acertadas, planes de mercadeo y publicidad y con un desarrollo de las habilidades de empresa en materia de la Administración de Operaciones.

El análisis de la implementación de la administración de operaciones en la organización debe conllevar el diseño de toda una metodología de aplicación, el gerente o administrador debe identificar las falencias existentes en cada uno de los procesos internos de esta manera al contar con una organización operativa y con las herramientas que brinda la Administración de operaciones se podría asegurar un resultado que brinde grandes oportunidades y beneficios a la empresa.

\section{REFERENCIAS}

[1] Saavedra, M. L. (2012). Una propuesta para la determinación de la competitividad en la pyme latinoamericana. Pensamiento \& Gestión, (33), 93-124.

[2] Saavedra, M. L., \& Saavedra, M. E. (2014). La PYME como generadora de empleo en México. Clio América; Santa Marta, 8(16), 153-172. https://doi.org/http://dx.doi.org/10.21676/238978 48.1350
[3] Cuadra, S. (2006). Nicaragua: enfoque estratégico de las PYMEs en un mundo globalizado. Encuentro, (74), 40-52.

[4] Rohvein, C., Paravie, D., Urrutia, S., Roark, G., Nunes, D., \& Ottogalli, D. (2013). Metodología de evaluación del nivel de competitividad de las pymes. Revista Ciencias Estratégicas, 21(29), 4968.

[5] Schroeder, R. G., \& Olaeta, R. D. L. P. (1992). Administración de operaciones: toma de decisiones en la función de operaciones (3a. ed, Vol. 17). McGraw-Hill México DF.

[6] Heizer, J., \& Render, B. (2004). Principios de administración de operaciones. Pearson Educación.

[7] Negrón, D. F. M. (2009). Administración de operaciones. Enfoque de administración de procesos de negocios. Cengage Learning Editores.

[8] Arnoletto, E. J. (2007). Administración de la producción como ventaja competitiva. Madrid, ES: B - EUMED.

[9] Guitart, L., \& Baraza, X. (2014). Dirección de operaciones: decisiones tácticas y estratégicas. Barcelona, ESPAÑA: Editorial UOC.

[10] Krajewski, L. J., \& Ritzman, L. P. (2000). Administración de operaciones: estrategia y análisis. Pearson Educación.

[11] Monks, J. G. (1988). Administración de operaciones. México, D.F., MX: McGraw-Hill Interamericana.

[12] García, S. M., Castorena, O. H., Escamilla, G. L., Guzmán, G. M., \& Florido, S. V. (2010). La influencia de la Administración de Operaciones en el rendimiento de la PyME. Investigación y Ciencia, 18(47), 66-74. 